\title{
RESEARCH
}

Open Access

\section{Co-existing cerebrovascular atherosclerosis predicts subsequent vascular event: a multi-contrast cardiovascular magnetic resonance imaging study}

Jin $\mathrm{Li}^{1,2+}$, Dongye $\mathrm{Li}^{2,3,4+}$, Dandan Yang ${ }^{4}$, Ran Huo ${ }^{5}$, Xiaoyi Chen ${ }^{6}$, Yilan $\mathrm{Xu}^{7}$, Wei Dai ${ }^{8}$, Dan Zhou ${ }^{1}$ and Xihai Zhao $2^{2^{*}}$

\begin{abstract}
Background: It is still unknown that whether co-existing intracranial stenosis and extracranial carotid vulnerable plaques have higher predictive value for subsequent vascular events. This study aimed to determine the relationship between co-existing extracranial carotid vulnerable plaques and intracranial stenosis and subsequent vascular events utilizing cardiovascular magnetic resonance (CMR) vessel wall imaging.
\end{abstract}

Methods: Patients who had recent cerebrovascular symptoms in anterior circulation $(<2$ weeks) were consecutively enrolled and underwent multi-contrast CMR vessel wall imaging for extracranial carotid arteries and 3D time-of flight CMR angiography for intracranial arteries at baseline. After baseline examination, all patients were followed-up for at least 1 year to determined recurrence of vascular events. The co-existing cerebrovascular atherosclerosis was defined as presence of both intracranial artery stenosis and at least one the following measures of extracranial artery atherosclerosis: plaque, calcification, lipid-rich necrotic core (LRNC), or intraplaque hemorrhage. Univariate and multivariate Cox regressions were used to calculate the hazard ratio (HR) and corresponding 95\% confidence interval (Cl) of co-existing plaques in predicting subsequent vascular events.

Results: In total, 150 patients (mean age: $61.8 \pm 11.9$ years; 109 males) were recruited. During the median follow-up time of 12.1 months, 41 (27.3\%) patients experienced vascular events. Co-existing intracranial artery stenosis and extracranial carotid plaque $(\mathrm{HR}, 3.57 ; 95 \% \mathrm{Cl}, 1.63-7.82 ; P=0.001)$ and co-existing intracranial artery stenosis and extracranial carotid LRNC $(H R, 4.47 ; 95 \% \mathrm{Cl}, 2.15-9.27 ; P<0.001)$ were significantly associated with subsequent vascular events, respectively. After adjusted for confounding factors and carotid stenosis, these associations remained statistically significant $(\mathrm{HR}, 5.12 ; 95 \% \mathrm{Cl}, 1.36-19.24 ; P=0.016$ and $\mathrm{HR}, 8.12 ; 95 \% \mathrm{Cl}, 2.41-27.31 ; P=0.001$, respectively).

Conclusions: The co-existing cerebrovascular atherosclerotic diseases, particularly co-existing carotid lipid-rich necrotic core and intracranial stenosis, are independent predictors for subsequent vascular events.

Keywords: Cerebrovascular, Atherosclerosis, Cardiovascular magnetic resonance imaging, Stroke, Acute coronary syndrome

\footnotetext{
* Correspondence: xihaizhao@tsinghua.edu.cn

${ }^{\dagger}$ Jin Li and Dongye Li contributed equally to this work.

${ }^{2}$ Center for Biomedical Imaging Research, Department of Biomedical

Engineering, Tsinghua University School of Medicine, Haidian District, Beijing

100084, China

Full list of author information is available at the end of the article
}

(c) The Author(s). 2020 Open Access This article is distributed under the terms of the Creative Commons Attribution 4.0 International License (http://creativecommons.org/licenses/by/4.0/), which permits unrestricted use, distribution, and reproduction in any medium, provided you give appropriate credit to the original author(s) and the source, provide a link to the Creative Commons license, and indicate if changes were made. The Creative Commons Public Domain Dedication waiver (http://creativecommons.org/publicdomain/zero/1.0/) applies to the data made available in this article, unless otherwise stated. 


\section{Introduction}

It has been shown that vulnerable atherosclerotic plaque is the major cause of ischemic stroke or acute coronary syndrome [1-3]. As a systematic disease, atherosclerotic disease commonly affects multiple vascular beds. The presence of atherosclerotic diseases in multiple vascular beds is usually defined as co-exiting plaques which represent the systemic burden of atherosclerosis $[4,5]$. Previous studies demonstrated that co-existing intracranial and extracranial atherosclerotic plaques had stronger predictive value for future vascular events compared with atherosclerosis in single vascular bed [6, 7]. However, the assessment of co-existing plaques in most of previous studies was focused on either the presence of plaques or measuring luminal stenosis. It is still unknown that whether coexisting intracranial stenosis and extracranial carotid vulnerable plaques have higher predictive value for subsequent vascular events. We hypothesized that co-existing carotid vulnerable plaque and intracranial stenosis may have stronger predictive value than each measurement alone for subsequent vascular events.

The co-existing intracranial and extracranial plaques can be characterized by multiple noninvasive imaging modalities, such as computed tomographic angiography (CTA) and cardiovascular magnetic resonance (CMR) angiography (CMRA) in clinical settings. However, both CTA and CMRA techniques could not provide the key compositional features of vulnerable plaques, such as lipid-rich necrotic core (LRNC) and intraplaque hemorrhage (IPH). This is because that these imaging modalities lack of vessel information. In contrast, it is well established that CMR vessel wall imaging is capable of accurately characterizing vulnerable plaque features in carotid arteries validated by histology [8].

This study aimed to determine the relationship between co-existing extracranial carotid vulnerable plaques and intracranial stenosis and subsequent vascular events utilizing CMR vessel wall imaging.

\section{Methods}

\section{Study population}

In this prospective study, patients who had recent cerebrovascular symptoms (ischemic stroke or transient ischemic attack [TIA] in the anterior circulation $<2$ weeks) were consecutively enrolled and underwent multi-contrast CMR vessel wall imaging for extracranial carotid arteries and three dimensional time-of-flight (3D-TOF) CMRA for intracranial arteries at baseline. The exclusion criteria are as follows: (1) hemorrhagic stroke; (2) cardiogenic stroke; (3) other vascular diseases including dissection, vasculitis, and moyamoya disease; (4) cerebral tumor; (5) history of radiation therapy in the neck; (6) pregnancy; (7) patients with severe disturbance of consciousness (coma, etc); (8) claustrophobia or any contraindication to CMR examination. Baseline clinical information for all patients including age, sex, body mass index (BMI), history of smoking, diabetes, hypertension, hyperlipidemia, stroke, coronary heart disease, National Institutes of Health Stroke Scale (NIHSS) score, the levels for lipoproteins (high density lipoprotein, low density lipoprotein, total cholesterol, and triglyceride) and the blood pressure was collected from the medical records. The information on treatment of antihypertension, lipid-lowering, anticoagulation and antiplatelet was also recorded during follow-up. The study protocol was approved by institutional review board and written consent form was obtained from each participant.

\section{Imaging acquisition}

At baseline, extracranial carotid artery and intracranial artery CMR imaging was conducted for all the patients on a $3 \mathrm{~T}$ CMR scanner (Achieva TX, Philips Healthcare, Best, The Netherlands) with custom-designed 36-channel neurovascular coil. The extracranial carotid arteries were imaged by using multi-contrast vessel wall imaging protocol that includes T1-weighted (T1W), T2-weighted (T2W), 3DTOF, and magnetization-prepared rapid acquisition gradient echo (MP-RAGE) and intracranial arteries were imaged by acquiring 3D-TOF CMRA. The imaging parameters are detailed in Table 1 . In addition, a 3D motion sensitized driven equilibrium rapid gradient echo (3D-MERGE) sequence was acquired for extracranial carotid artery vessel wall imaging with the following parameters: fast field echo, repeat time / echo time $9.2 / 4.3 \mathrm{~ms}$, flip angle $6^{\circ}$, field of view $20 \times 16 \times 4 \mathrm{~cm}^{3}$; isotropic spatial resolution $0.8 \times 0.8 \times$ $0.8 \mathrm{~mm}^{3}$. A routine protocol, including $\mathrm{T} 1 \mathrm{w}$, fluid attenuated inversion recovery (FLAIR) and diffusion weighted imaging (DWI) sequences, was used to assess the cerebral infarct during follow-up.

\section{Follow-up and clinical outcome}

After CMR imaging at baseline, all patients were followed-up for at least 1 year. The clinical outcome was defined as any vascular event, such as ischemic stroke, TIA, or acute coronary syndrome. All patients were followed-up by telephone or routine medical outpatient clinic attendance and inquiring whether patients had experienced any vascular event in the past time. If patients did not respond to the follow-up, we will find their medical records and try to contact their relatives for more information about the patient's condition.

\section{Image analysis}

The CMR images were interpreted by two reviewers with $>3$ years' experience in vascular imaging using customdesigned software of CASCADE (University of Washington, Seattle, Washington, USA) with consensus blinded to clinical information. According to the sharpness and contrast between the vessel wall and the surrounding fat tissues by eyeballing, a 4-point scale was adopted: 1 , poor; 2 , marginal; 
Table 1 The parameters for carotid artery and intracranial artery CMR imaging

\begin{tabular}{|c|c|c|c|c|c|}
\hline & \multicolumn{5}{|c|}{ CMR imaging parameters } \\
\hline & \multicolumn{4}{|c|}{ Extracranial carotid artery } & \multirow{2}{*}{$\begin{array}{l}\text { Intracranial artery } \\
\text { TOF-CMRA }\end{array}$} \\
\hline & $\mathrm{T1W}$ & $\mathrm{T} 2 \mathrm{~W}$ & TOF & MP-RAGE & \\
\hline Sequence & TSE & TSE & FFE & FFE & FFE \\
\hline Black blood & QIR & MDIR & - & - & - \\
\hline $\mathrm{TR}, \mathrm{ms}$ & 800 & 4800 & 20 & 8.8 & 25 \\
\hline $\mathrm{TE}, \mathrm{ms}$ & 10 & 50 & 4.9 & 5.3 & 3.5 \\
\hline Flip angle, deg & $90^{\circ}$ & $90^{\circ}$ & $20^{\circ}$ & $15^{\circ}$ & $20^{\circ}$ \\
\hline $\mathrm{FOV}, \mathrm{mm}$ & $140 \times 140$ & $140 \times 140$ & $140 \times 140$ & $140 \times 140$ & $240 \times 240$ \\
\hline In-plane resolution, $\mathrm{mm}^{2}$ & $0.5 \times 0.5$ & $0.5 \times 0.5$ & $0.5 \times 0.5$ & $0.5 \times 0.5$ & $0.7 \times 0.7$ \\
\hline Slice thickness, mm & 2 & 2 & 1 & 1 & 1.4 \\
\hline
\end{tabular}

TOF time-of-flight, MP-RAGE Magnetization Prepared Gradient Recalled Echo, CMRA cardiovascular magnetic resonance angiography, TSE turbo spin echo, FFE fast field echo, QIR quadruple inversion recovery, MDIR multislice double inversion recovery. TR repeat time, TE echo time, FOV field of view, TIW T1 weighted, T2W T2 weighted

3, good; 4, excellent. The CMR images with image scale $\geq 2$ were interpreted. Carotid plaque was defined as intimamedia thickness $\geq 1.5 \mathrm{~mm}$ on ultrasound, eccentric wall thickening or having any plaque compositions such as calcification, LRNC, or IPH on CMR. The boundaries of carotid artery lumen and wall were traced manually and the maximum wall thickness (Max WT) which was a representative of plaque burden was measured. For patients with ischemic stroke, the carotid artery which is responsible for the symptoms was considered as index side and included in the statistical analysis. For patients with TIA, the index artery was defined as lesions with high-risk plaque feature, including large LRNC (occupied $>40 \%$ of wall area), IPH and fibrous cap rupture, or larger Max WT bilaterally when there was no high-risk feature. The intracranial artery stenosis was measured on 3D-TOF CMRA images, in the following segments of arteries: intracranial internal carotid arteries, anterior cerebral arteries (A1) and middle cerebral arteries (M1). The presence or absence of each plaque composition, such as calcification, LRNC, IPH, was determined by utilizing published criteria $[9,10]$. The luminal stenosis was measured using WASID [11] criteria for intracranial arteries and North American Symptomatic Carotid Endarterectomy Trial (NASCET) [12] criteria for extracranial carotid arteries, respectively. The intracranial artery stenosis was categorized into the following categories: $(1)<50 \%$; and (2) $\geq 50 \%$. The extracranial carotid artery stenosis was categorized into the following categories: (1) <50\%; (2) $50-69 \%$; and (3) $\geq 70 \%$. The carotid culprit lesion was defined as atherosclerotic plaque with $>50 \%$ stenosis or high-risk feature, such as large LRNC (occupied $>40 \%$ of wall area), IPH and fibrous cap rupture. For intracranial arteries, the culprit lesion was defined as lesion with $>50 \%$ stenosis. The coexisting cerebrovascular atherosclerotic diseases were defined as presence of both intracranial artery stenosis and at least one the following measures of extracranial artery atherosclerosis: plaque, calcification, LRNC, or IPH. The presence or absence of cerebral infarct of anterior circulation was assessed on DWI or FLAIR images during followup. The presence or absence of acute coronary syndrome was determined by myocardial enzyme detection or coronary arteriography during follow-up.

\section{Reproducibility}

Twenty patients were randomly selected for testing the intra-observer and inter-observer reproducibility in identifying presence of plaque, calcification, LRNC, IPH and measuring Max WT and stenosis at extracranial carotid artery. The intracranial artery stenosis was also measured for all 20 patients. A time interval of 3 months was set for determining the intra-observer reproducibility to minimize the bias of memory.

\section{Statistical analysis}

The continuous variables were described as mean and standard deviation and the categorical variables were presented as frequency. Baseline clinical characteristics, carotid plaque features and intracranial artery stenosis were compared between patients with and without VEs using independent $t$ test, Mann-Whitney $\mathrm{U}$ test, or Chi-square when appropriate. In analyzing the predictors of the clinical outcome, univariate and multivariate Cox proportional hazards regression functions were used to calculate hazard ratio (HR) and the corresponding 95\% confidence interval (CI) of possible determinants of vascular events, taking the time variable into consideration. The Kaplan-Meier productlimit method was used to estimate cumulative event-free rates in subgroups for graphical display depending on the presence of co-existing plaques. Cohen's kappa was utilized to determine the inter-observer and intra-observer agreement in identification of presence of carotid plaque, calcification, LRNC and IPH. The intra-class correlation coefficient (ICC) was calculated to assess the inter-observer and intra-observer agreements in quantitatively measuring 
the intracranial artery stenosis, carotid artery stenosis and Max WT. The $P$ value $<0.05$ was considered as statistically significant. The statistical analyses were carried out using SPSS (version 19, Statistical Package for the Social Sciences, International Business Machines, Inc., Armonk, New York, USA).

\section{Results}

Of 173 recruited patients, 23 were excluded including 7 with poor image quality, 8 with loss of follow-up and 8 with carotid surgery during follow-up. Among the remaining 150 patients [61.8 \pm 11.9 years; $109(72.7 \%)$ males], 107 (71.3\%) had ischemic stroke in anterior circulation including 56 (37.3\%) patients with acute cerebral infarction, 51 (34.0\%) patients with acute lacunar infarction and 43 (28.7\%) patients with TIA. Among the 150 patients who suffered from cerebrovascular symptoms at baseline, 99 (66.0\%) had culprit lesions in extracranial carotid arteries, $48(32.0 \%)$ had culprit lesions in intracranial arteries and the culprit lesions were uncertain in $3(2.0 \%)$ patients. The clinical data are detailed in Table 2. Patients with subsequent vascular events had significantly greater age (64.9 \pm
10.4 vs. $60.6 \pm 12.2$ years old, $P=0.047)$ and NIHSS score (5.6 \pm 4.3 vs. $3.3 \pm 3.0, P=0.003)$ compared to those without subsequent vascular events. No significant differences can be observed in other clinical characteristics between these two patient groups (all $P>0.05$ ).

\section{Baseline characteristics of cerebrovascular atherosclerosis on CMR imaging}

Of 150 patients, 38 (25.3\%) had stenosis < 50\%, 4 (2.7\%) had stenosis in the range of 50-69\%, and 15 (10.0\%) had stenosis $\geq 70 \%$ in extracranial carotid arteries, respectively. In this study population, the prevalence of plaque, calcification, LRNC and IPH was 88.0\% (132/150), 44.0\% $(66 / 150), 66.0 \%(99 / 150)$, and $17.3 \%(26 / 150)$ in extracranial carotid arteries, respectively. In intracranial arteries, 48 (32.0\%) patients had stenosis $<50 \%$ and 48 (32.0\%) patients had stenosis $\geq 50 \%$, respectively. The distribution of intracranial artery stenosis is detailed in Additional file 1: Table S1. The comparison results of cerebrovascular atherosclerosis between patients with and without subsequent vascular events are summarized in Table 3. Patients with subsequent vascular events had

Table 2 Baseline and follow-up clinical characteristics of the study population $(n=150)$

\begin{tabular}{|c|c|c|c|}
\hline & \multicolumn{2}{|l|}{ Mean $\pm \mathrm{SD}$, or $n(\%)$} & \multirow[b]{2}{*}{$P$ value } \\
\hline & Patients with vascular events $(n=41)$ & Patients without vascular events $(n=109)$ & \\
\hline \multicolumn{4}{|l|}{ Baseline clinical characteristics } \\
\hline Age, years & $64.9 \pm 10.4$ & $60.6 \pm 12.2$ & 0.047 \\
\hline Sex, male & $28(68.3)$ & $81(74.3)$ & 0.461 \\
\hline $\mathrm{BMI}, \mathrm{kg} / \mathrm{m}^{2}$ & $25.6 \pm 3.3$ & $25.5 \pm 3.4$ & 0.868 \\
\hline History of smoking & $24(58.5)$ & $67(61.5)$ & 0.743 \\
\hline History of hypertension & $28(68.3)$ & $79(72.5)$ & 0.614 \\
\hline History of diabetes & $7(17.1)$ & $35(32.1)$ & 0.068 \\
\hline History of hyperlipidemia & $16(39.0)$ & $59(54.1)$ & 0.099 \\
\hline History of coronary heart disease & $5(12.2)$ & $12(11.0)$ & 0.839 \\
\hline History of stroke & $20(48.8)$ & $38(34.9)$ & 0.119 \\
\hline Systolic blood pressure, $\mathrm{mm} \mathrm{Hg}$ & $147.6 \pm 23.2$ & $144.7 \pm 24.0$ & 0.223 \\
\hline Diastolic blood pressure, mm Hg & $88.6 \pm 13.5$ & $86.6 \pm 16.2$ & 0.236 \\
\hline $\mathrm{HDL}, \mathrm{mmol} / \mathrm{L}$ & $1.1 \pm 0.3$ & $1.2 \pm 0.6$ & 0.374 \\
\hline $\mathrm{LDL}, \mathrm{mmol} / \mathrm{L}$ & $2.5 \pm 1.4$ & $2.9 \pm 1.4$ & 0.096 \\
\hline Total cholesterol, mmol/L & $4.2 \pm 1.3$ & $4.4 \pm 1.2$ & 0.398 \\
\hline Triglycerides, mmol/L & $1.7 \pm 1.4$ & $1.7 \pm 1.0$ & 0.646 \\
\hline NIHSS score & $5.6 \pm 4.3$ & $3.3 \pm 3.0$ & 0.003 \\
\hline \multicolumn{4}{|l|}{ Drug treatment during follow-up } \\
\hline Antihypertension & $17(41.5)$ & $49(45.0)$ & 1.000 \\
\hline Lipid-lowering & $33(80.5)$ & $86(78.9)$ & 0.203 \\
\hline Anticoagulation & $22(53.7)$ & $63(57.8)$ & 0.064 \\
\hline Antiplatelet & $21(51.2)$ & 39 (35.8) & 0.368 \\
\hline
\end{tabular}


Table 3 Characteristics of intracranial artery and extracranial carotid artery atherosclerosis

\begin{tabular}{|c|c|c|c|}
\hline & \multicolumn{2}{|l|}{ Mean $\pm S D$, or $n(\%)$} & \multirow{2}{*}{$\begin{array}{l}\mathrm{P} \\
\text { value }\end{array}$} \\
\hline & Patients with vascular events $(n=41)$ & Patients without vascular events $(n=109)$ & \\
\hline \multicolumn{4}{|l|}{ Extracranial carotid artery } \\
\hline Maximum wall thickness, mm & $3.3 \pm 1.7$ & $3.0 \pm 1.4$ & 0.316 \\
\hline \multicolumn{4}{|l|}{ Presence of stenosis } \\
\hline Intracranial stenosis & $33(80.5)$ & $63(57.8)$ & 0.010 \\
\hline Extracranial carotid stenosis & $17(41.5)$ & $40(36.7)$ & 0.592 \\
\hline \multicolumn{4}{|c|}{ Presence of extracranial carotid plaque components } \\
\hline Calcification & $22(53.7)$ & $44(40.4)$ & 0.144 \\
\hline Lipid-rich necrotic core & $34(82.9)$ & $65(59.6)$ & 0.007 \\
\hline Intraplaque hemorrhage & $9(22.0)$ & $17(15.6)$ & 0.359 \\
\hline
\end{tabular}

significantly greater prevalence of intracranial stenosis $(80.5 \%$ vs. $57.8 \%, P=0.010)$ and extracranial carotid LRNC $(82.9 \%$ vs. $59.6 \%, P=0.007)$ compared to those without subsequent vascular events. No significant differences can be found in prevalence of other plaque compositions (all $P>0.05$ ).

\section{Association between co-existing plaques and subsequent vascular events}

Of the 150 patients, 96 (64.0\%) had intracranial stenosis, of which 89 (59.3\%), 45 (30.0\%), 70 (46.7\%), and 19 (12.7\%) had co-existing plaque, calcification, LRNC, and IPH in carotid arteries, respectively. After baseline examination, some patients with carotid endarterectomy $(n=8)$ were excluded from our study and the remaining patients $(n=$ 150) received the medical treatment during follow-up. During the median follow-up time of 12.1 months, 41 (27.3\%) patients were suffered from subsequent vascular events including 15 (10.0\%) patients with recurrent ischemic stroke diagnosed by CMR and 26 (17.3\%) patients with recurrent vascular events determined by medical records during follow-up. Of 41 patients with recurrent vascular events, 17 had ischemic strokes in the territory of anterior circulation (11 acute cerebral infarcts and 6 acute lacunar infarcts), 19 had TIAs, and 5 had acute coronary syndromes. Table 4 presented the results on Cox regression analysis. Univariate Cox regression analysis showed that co-existing intracranial stenosis and carotid plaque (HR, 3.57; 95\% CI, 1.63-7.82; $P=0.001$ ) and LRNC (HR, 4.47; 95\% CI, 2.15-9.27; $P<0.001)$ were significantly associated with subsequent vascular events. After adjusted for baseline confounding factors including age, sex, BMI, history of stroke, diabetes, hypertension, hyperlipidemia, coronary heart disease, smoking, NIHSS, carotid stenosis and treatment procedures including antihypertension, lipidlowering, anticoagulation and antiplatelet during followup, these associations remained statistically significant (coexisting intracranial stenosis and carotid plaque: $\mathrm{HR}=$ 5.12, 95\% CI 1.36-19.24, $P=0.016$; co-existing intracranial stenosis and carotid LRNC: $\mathrm{HR}=8.12,95 \%$ CI 2.41-27.31, $P=0.001)$. The subsequent vascular events were not significantly associated with co-existing intracranial stenosis and carotid IPH and calcification (all $P>0.05$ ). Since only few cases did not have carotid plaques, the HR value of presence of extracranial carotid plaque for predicting subsequent vascular events was not calculated due to the potential issue of overfitting. The prevalence of cumulative vascular events for patients with co-existing intracranial artery stenosis and extracranial carotid plaque at 5, 10, 15 and 20 months was $4.0 \%(6 / 150), 7.3 \%(11 / 150), 21.3 \%$ $(32 / 150)$ and $22.0 \%(33 / 150)$, respectively. The prevalence of cumulative vascular events for patients with co-existing intracranial artery stenosis and extracranial carotid LRNC at $5,10,15$ and 18 months was $4.0 \%(6 / 150), 6.7 \%(10 /$ $150), 20.0 \%(30 / 150)$ and $20.0 \%(30 / 150)$, respectively. Kaplan-Meier curves for the incidence of subsequent vascular events showed that event-free survival was significantly higher for patients in the nonco-existing intracranial artery stenosis and extracranial carotid plaque group than ones in the co-existing intracranial artery stenosis and extracranial carotid plaque group $(P=0.001$; Fig. 1a). KaplanMeier curves for the incidence of subsequent vascular events showed that event-free survival was significantly higher for patients in the nonco-existing intracranial artery stenosis and extracranial carotid LRNC group than ones in the co-existing intracranial artery stenosis and extracranial carotid LRNC group $(P<0.001$; Fig. $1 b)$. Figure 2 is an example for patient who had co-existing intracranial and extracranial carotid LRNC developed subsequent ischemic stroke after one-year follow-up.

\section{Reproducibility}

For the intra-observer agreement in identification of the presence of carotid plaque, calcification, LRNC and IPH, the kappa value was $1.00,0.78,0.80$ and 0.89 (all $P<$ 0.001 ), respectively. For the inter-observer agreement in assessing of the presence of carotid plaque, calcification, LRNC and IPH, the kappa value was 1.00, 0.87, 0.80 and 
Table 4 Cox regression hazard models of risk factors for vascular events

\begin{tabular}{|c|c|c|c|c|c|c|}
\hline & \multicolumn{6}{|c|}{ Vascular events } \\
\hline & \multicolumn{3}{|c|}{ Univariate Regression } & \multicolumn{3}{|c|}{ Multivariate Regression* } \\
\hline & $\overline{\mathrm{HR}}$ & $95 \% \mathrm{Cl}$ & $P$ value & $\mathrm{HR}$ & $95 \% \mathrm{Cl}$ & $P$ value \\
\hline \multicolumn{7}{|l|}{ Extracranial carotid artery } \\
\hline Maximum wall thickness, mm & 1.06 & $0.88-1.28$ & 0.542 & 0.91 & $0.67-1.23$ & 0.526 \\
\hline Presence of calcification & 1.40 & $0.76-2.60$ & 0.280 & 1.04 & $0.32-3.33$ & 0.954 \\
\hline Presence of LRNC & 3.65 & $1.60-8.35$ & 0.002 & 4.26 & $0.96-18.80$ & 0.056 \\
\hline Presence of IPH & 1.25 & $0.60-2.64$ & 0.552 & 0.57 & $0.09-3.60$ & 0.552 \\
\hline Presence of stenosis & 1.16 & $0.62-2.16$ & 0.647 & - & - & - \\
\hline \multicolumn{7}{|l|}{ Intracranial artery } \\
\hline Presence of stenosis & 2.73 & $1.25-5.97$ & 0.012 & 5.95 & $0.97-36.38$ & 0.054 \\
\hline \multicolumn{7}{|l|}{ Co-existing plaques } \\
\hline Intracranial stenosis and extracranial carotid plaque & 3.57 & $1.63-7.82$ & 0.001 & 5.12 & $1.36-19.24$ & 0.016 \\
\hline Intracranial stenosis and extracranial carotid calcification & 1.74 & $0.94-3.24$ & 0.078 & 2.95 & $0.76-11.42$ & 0.117 \\
\hline Intracranial stenosis and extracranial carotid LRNC & 4.47 & $2.15-9.27$ & $<0.001$ & 8.12 & $2.41-27.31$ & 0.001 \\
\hline Intracranial stenosis and extracranial carotid IPH & 1.67 & $0.77-3.63$ & 0.194 & 1.44 & $0.17-12.39$ & 0.738 \\
\hline
\end{tabular}

0.78 (all $P<0.001$ ), respectively. The intra-observer ICC of carotid Max WT, luminal stenosis and intracranial artery stenosis was 0.98 (95\% CI, 0.94-0.99, $P<0.001)$, 0.96 (95\% CI, 0.90-0.98, $P<0.001$ ) and 0.98 (95\% CI, 0.94-0.99, $P<0.001)$, respectively. The inter-observer ICC was 0.98 (95\% CI, 0.96-0.99, $P<0.001), 0.93$ (95\% CI, 0.84-0.97, $P<0.001$ ) and 0.98 (95\% CI, 0.94-0.99, $P<0.001)$ for carotid Max WT, luminal stenosis and intracranial artery stenosis, respectively.

\section{Discussion}

This study investigated the relationship between co-existing intracranial stenosis and extracranial vulnerable plaques and subsequent vascular events utilizing CMR vessel wall imaging. We found that the co-existing intracranial arteries stenosis and extracranial carotid plaque or LRNC determined by CMR vessel wall imaging was independently associated with subsequent vascular events. Our findings suggest that co-existing intracranial stenosis with extracranial carotid artery plaque vulnerable features, such as LRNC, have higher predictive value for occurrence of subsequent vascular events compared to the measurement in single vascular bed.

In the present study, $59.3 \%$ of symptomatic patients had co-existing extracranial carotid artery plaque and intracranial artery stenosis. Our findings are in line with previous reports with the prevalence of co-existing plaques ranging from 42.2 to $64 \%$ in symptomatic patients $[4,5,13]$. Different from previous studies, $\mathrm{Xu}$ et al. [10] utilized CMR vessel wall imaging to detect atherosclerotic disease and found that the prevalence of co-existing intracranial and extracranial plaque was $77.6 \%$ in symptomatic patients. In the present study, the presence of extracranial carotid plaque was also determined by CMR vessel wall imaging. It is well established that CMR vessel wall imaging can yield more lesion detection compared with angiographic imaging approach because it provides direct information of the lesions in the arterial wall $[14,15]$. The prevalence of co-existing cerebrovascular atherosclerotic plaques in the present study was lower than that in Xu's study [10] which might be due to different study population. In Xu's study [10], all the patients had plaque in at least one side of carotid arteries. The presence of co-existing plaques in intra- and extra-cranial vascular beds further demonstrated that the atherosclerosis can develop systemically.

We found that the co-existing intracranial artery stenosis and extracranial carotid plaque determined by CMR vessel wall imaging was independently associated with subsequent vascular events. The co-existing intracranial and extracranial atherosclerosis may represent a systematic burden of atherosclerotic disease. Similar to our findings, previous studies also reported that patients with coexisting plaques had higher risk of cardiovascular or cerebrovascular events. A prospective study in which patients were followed-up up to 76 months by Man et al. [16] showed that the presence of concurrent intracranial and extracranial stenoses measured by CMRA was independent predictors of poor outcomes. Hoshino et al. [17] investigated the vascular prognosis for patients with acute ischemic stroke with intracranial and extracranial plaques after 4 years of follow-up and found that among patients with 

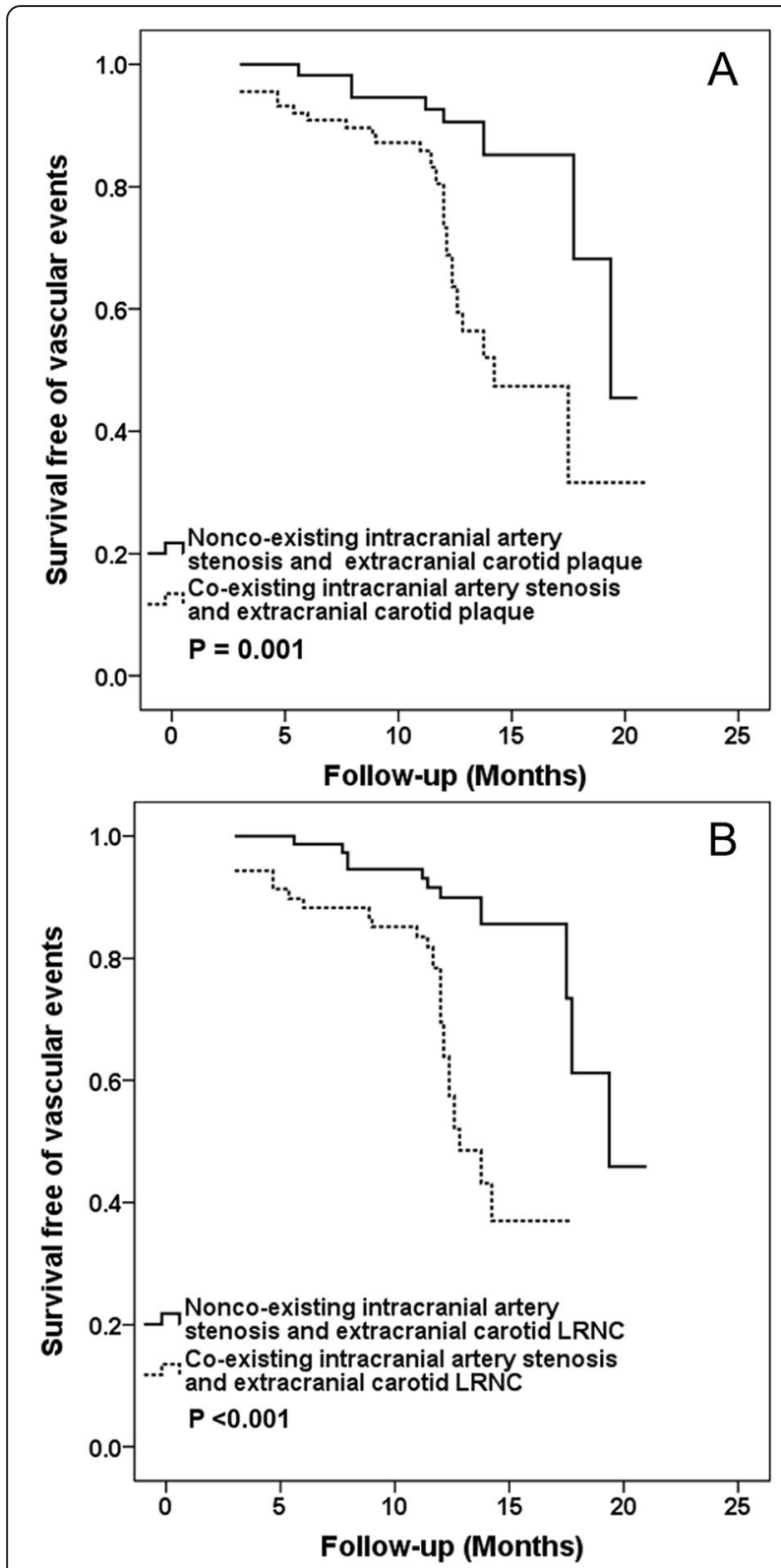

Fig. 1 Kaplan-Meier analysis of survival free of vascular eventsin coexisting diseases. Kaplan-Meier analysis of survival free of vascular events in the nonco-existing intracranial artery stenosis and extracranial carotid plaque and co-existing intracranial artery stenosis and extracranial carotid plaque with up to 21 months follow-up (a). Kaplan-Meier analysis of survival free of vascular events in the nonco-existing intracranial artery stenosis and extracranial carotid lipid rich necrotic core (LRNC) and co-existing intracranial artery stenosis and extracranial carotid LRNC with up to 21 months followup (b). The X-axis represents the time of follow-up in months. The $Y$ axis represents the proportion of patients who were survival free of vascular events

intracranial atherosclerosis, concurrent stenosis in the extracranial carotid artery $(23.4 \%$ vs. $9.0 \% ; P=0.08$; adjusted $\mathrm{HR}=2.12$ ) increased the major adverse cardiovascular events risk. These findings indicate that the co-existing intracranial and extracranial plaques can predict future vascular events.

The co-existing intracranial artery stenosis and extracranial carotid LRNC were independently associated with subsequent vascular events. This study is one of the first to investigate the predictive value of co-existing carotid vulnerable plaque and intracranial stenosis for future vascular events. The finding is expected because the association between carotid LRNC and cerebrovascular events has been well evidenced in previous studies. Takaya et al. [18] demonstrated that among patients who initially had an asymptomatic 50 to $79 \%$ carotid stenosis, larger maximum percentage of LRNC (HR for 10\% increase, 1.6; $P=$ 0.004) by CMR was associated with the occurrence of subsequent cerebrovascular events. Another CMR study by Kwee et al. [19] claimed that the presence of LRNC (HR, 3.20; 95\% CI, 1.08-9.50; $P=0.036$ ) was associated with the recurrence of clinical cerebrovascular ischemic events in TIA and stroke patients with carotid atherosclerosis. Investigators also found that there was significant correlation between carotid LRNC and cardiovascular events. Zavodni et al. [20] showed that a lipid core at CMR imaging confers increased risk for subsequent cardiovascular events in asymptomatic individuals. Sun et al. [21] reported that high lipid content (HR per one-standarddeviation increase in \% lipid core volume: $1.57, P=0.002$ ) in carotid plaques was strongly associated with the cardiovascular outcomes. The LRNC within atherosclerotic plaque, particularly its size, has been believed to be a key feature of vulnerable plaque [22, 23]. The increasement of the LRNC size may be contributed to the changes of cholesterol from the liquid to solid crystal state and will stimulate fibrous cap extension and thinning or even rupture [24]. In the presence study, we used CMR vessel wall imaging to identify not only LRNC but also IPH. However, we did not find significant correlation of co-existing intracranial stenosis and extracranial carotid IPH with vascular events, though this compositional feature is associated with plaque vulnerability [22]. The potential reason may be the smaller number of co-existing IPH $(n=26)$ in this study population. Our findings indicate that the assessment of co-existing intracranial and extracranial atherosclerosis needs to not only pay attention to presence of plaque but also characterize plaque compositional features, particularly lipid-rich components. Our findings suggest that patients with co-existing lesions may have higher risk of developing future vascular events and intense intervention medication may be warranted for these patients.

\section{Limitations}

The present study has several limitations. First, the intracranial atherosclerosis was identified by TOF CMRA that lacks of vessel wall information. TOF CMRA may yield underestimation of intracranial plaques when there is positive 


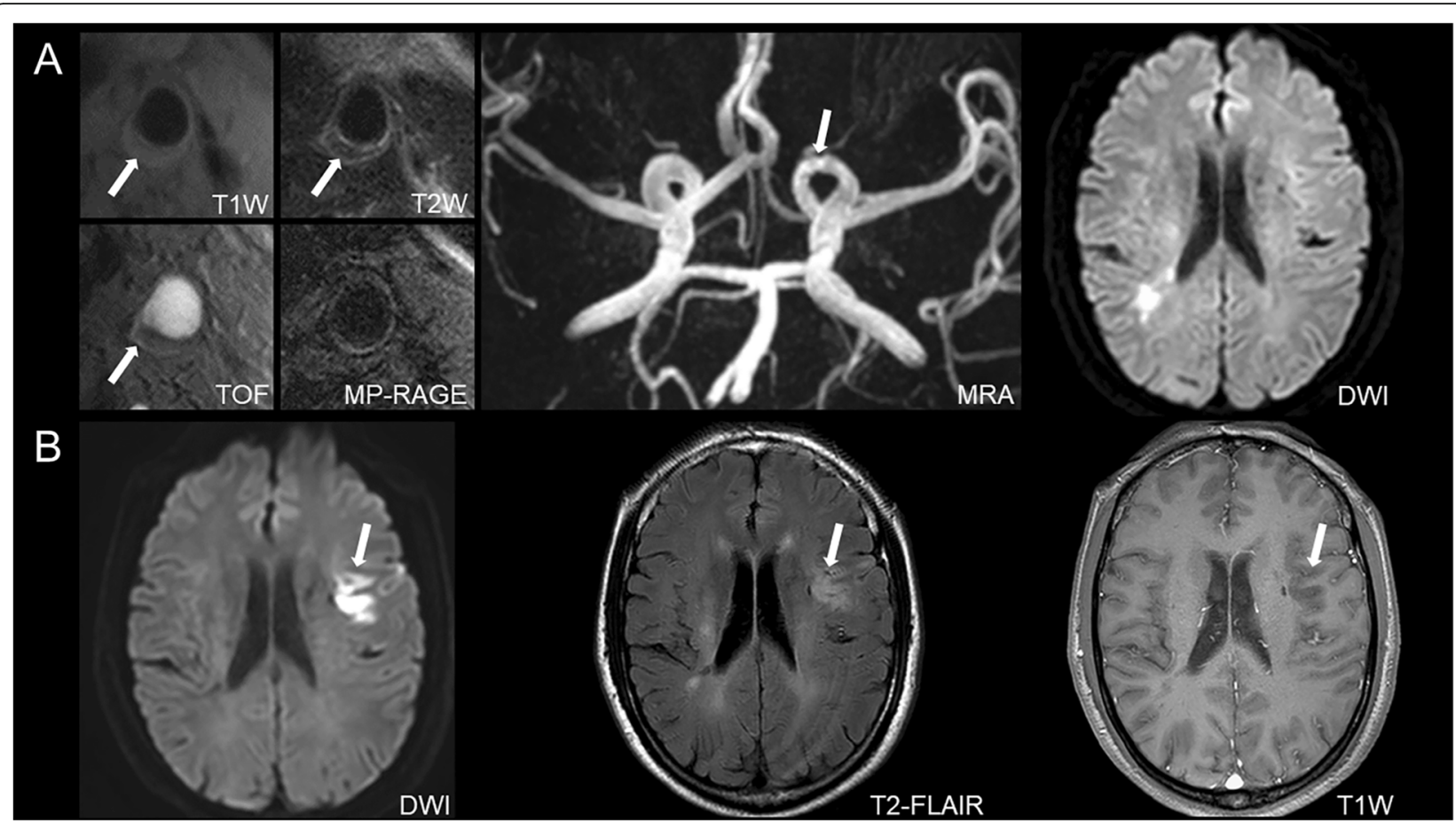

Fig. 2 Patient with co-existing intracranial artery stenosis and extracranial carotid LRNC developed recurrent stroke. An example for a 63 years old male patient who had atherosclerotic plaque with lipid-rich necrotic core in the left extracranial carotid artery bifurcation (white arrows on A $[11$ weighted (T1W), T2 weighted (T2W) and time-of-flight (TOF)]) and intracranial artery stenosis (white arrow on A [MRA]). After a year of follow-up, a new acute infarction was detected in the left hemisphere (white arrows on B [diffusion weighted imaging (DWI), T2-FLAIR and T1w])

remodeling in the vessel wall [25]. Future studies utilizing vessel wall imaging to characterize plaque features of intracranial arteries are warranted. Second, the follow-up time was short and the usefulness of co-existing plaque in predicting long-term outcomes cannot be determined. Third, in the present study, the size of the intraplaque compositions of co-existing intracranial and extracranial artery atherosclerosis was not assessed. Previous evidences showed that the size of LRNC $[18,21]$ and IPH [18] was associated with future vascular events. Fourth, the longitudinal coverage of current carotid imaging protocol is $32 \mathrm{~mm}$ centered to carotid bifurcation. This coverage is limited for atherosclerotic lesions occurring in more proximal or distal segments of carotid arteries. The 3D vessel wall imaging techniques [26] with larger longitudinal coverage are suggested to be utilized in future studies. Finally, the HR value of extracranial carotid plaque in predicting vascular eventswas not analyzed because atherosclerotic plaques were present in most of the cases of our study population. Future studies will increase the sample size to enhance such statistical power.

\section{Conclusions}

The co-existing cerebrovascular atherosclerotic diseases, particularly co-existing carotid lipid-rich necrotic core and intracranial stenosis, are independent predictors for subsequent vascular events. Our findings suggest that it is valuable to characterize the compositional features of coexisting intracranial and extracranial carotid atherosclerosis in stratifying the risk of subsequent vascular events.

\section{Supplementary information}

Supplementary information accompanies this paper at https://doi.org/10. 1186/s12968-019-0596-6.

Additional file $\mathbf{1}$ Table S1. The distribution of intracranial artery stenosis.

\section{Abbreviations}

BMI: Body mass index; Cl: Confidence interval; CMR: Cardiovascular magnetic resonance; CMRA: Cardiovascular magnetic resonance angiography; CTA: Computed tomographic angiography; DWl: Diffusion weighted imaging; FLAIR: Fluid attenuated inversion recovery; HR: Hazard ratio; ICC: Intra-class correlation coefficient; IPH: Intraplaque hemorrhage; LRNC: Lipid-rich necrotic core; Max WT: Maximum wall thickness; MERGE: Motion sensitized driven Equilibrium prepared Rapid Gradient Echo; MP-RAGE: Magnetization-prepared rapid acquisition gradient echo; NASCET: North American Symptomatic Carotid Endarterectomy Trial; T1W: T1 weighted; T2W: T2 weighted; TIA: Transient ischemic attack; TOF: Time-offlight

\section{Acknowledgements}

Not applicable.

\section{Authors' contributions}

J.L. and D.L. interpreted data, performed statistical analysis and drafted the manuscript. D.Y., R.H., X.C. and Y.X. analyzed the data. W.D., D.L., and D.Z. acquired the data. X.Z. conceived the overall study, handled funding and 
supervision, and made critical revision of the manuscript. All authors read and approved the final manuscript.

\section{Funding}

This work was supported by National Natural Science Foundation of China (81771825), Beijing Municipal Science and Technology Commission (D171100003017003), and Ministry of Science and Technology of China (2017YFC1307904).

\section{Availability of data and materials}

The datasets used and/or analyzed during the current study are available from the corresponding author [X.Z.] on reasonable request. The data are not publicly available due to them containing information that could compromise research participant privacy/consent.

\section{Ethics approval and consent to participate}

The study protocol was approved by institutional review board of Tsinghua University School of Medicine and the written consent forms were obtained from all the subjects prior to the initiation of this study.

\section{Consent for publication}

Not applicable.

\section{Competing interests}

The authors declare that they have no competing interests.

\section{Author details}

${ }^{1}$ Department of Radiology, The Affiliated BenQ Hospital of Nanjing Medical University, Nanjing, China. ${ }^{2}$ Center for Biomedical Imaging Research, Department of Biomedical Engineering, Tsinghua University School of Medicine, Haidian District, Beijing 100084, China. ${ }^{3}$ Department of Radiology, Sun Yat-Sen Memorial Hospital, Sun Yat-Sen University, Guangzhou, China. ${ }^{4}$ Beijing Institute of Brain Disorders, Capital Medical University, Beijing, China. ${ }^{5}$ Department of Radiology, Peking University Third Hospital, Beijing, China. ${ }^{6}$ Department of Radiology, Beijing Geriatric Hospital, Beijing, China. ${ }^{7}$ Department of Radiology, Beijing Tsinghua Changgung Hospital, School of Clinical Medicine, Tsinghua University, Beijing, China. ${ }^{8}$ Department of Neurology, Fourth Medical Center of Chinese PLA General Hospital, Beijing, China.

Received: 5 July 2019 Accepted: 20 December 2019

Published online: 13 January 2020

\section{References}

1. Qureshi Al, Caplan LR. Intracranial atherosclerosis. Lancet. 2014;383:984-98.

2. Gupta A, Baradaran H, Schweitzer AD, Kamel H, Pandya A, Delgado D, et al. Carotid plaque MRI and stroke risk: a systematic review and meta-analysis. Stroke. 2013:44:3071-7.

3. Boateng S, Sanborn T. Acute myocardial infarction. Dis Mon. 2013;59:83-96.

4. Liu HM, Tu YK, Yip PK, Su CT. Evaluation of intracranial and extracranial carotid steno-occlusive diseases in Taiwan Chinese patients with MR angiography: preliminary experience. Stroke. 1996;27:650-3.

5. Yang F, Liu L, Li M, Li M, Yin Q, Guo R, et al. Pattern of cerebrovascular atherosclerotic stenosis in older Chinese patients with stroke. J Clin Neurosci. 2013;20:979-83.

6. Heldner MR, Li L, Lovett NG, Kubiak MM, Lyons S, Rothwell PM, et al. Longterm prognosis of patients with transient ischemic attack or stroke and symptomatic vascular disease in multiple arterial beds. Stroke. 2018:49:1639-46.

7. Li D, Dai W, Cai Y, Han Y, Yao G, Chen H, et al. Atherosclerosis in strokerelated vascular beds and stroke risk: a 3-D MR vessel wall imaging study. Ann Clin Transl Neurol. 2018;5:1599-610.

8. Cai JM, Hatsukami TS, Ferguson MS, Small R, Polissar NL, Yuan C. Classification of human carotid atherosclerotic lesions with in vivo multicontrast magnetic resonance imaging. Circulation. 2002;106:1368-73.

9. Yuan C, Mitsumori LM, Ferguson MS, Polissar NL, Echelard D, Ortiz G, et al. In vivo accuracy of multispectral magnetic resonance imaging for identifying lipid-rich necrotic cores and intraplaque hemorrhage in advanced human carotid plaques. Circulation. 2001;104:2051-6.

10. Xu Y, Yuan C, Zhou Z, He L, Mi D, Li R, et al. Co-existing intracranial and extracranial carotid artery atherosclerotic plaques and recurrent stroke risk: a three-dimensional multicontrast cardiovascular magnetic resonance study. J Cardiovasc Magn Reson. 2016;18:90.

11. Chimowitz MI, Lynn MJ, Howlett-Smith H, Stern BJ, Hertzberg VS, Frankel $M R$, et al. Comparison of warfarin and aspirin for symptomatic intracranial arterial stenosis. N Engl J Med. 2005;352:1305-16.

12. Inzitari $D$, Eliasziw M, Gates $P$, Sharpe BL, Chan RK, Meldrum HE, et al. The causes and risk of stroke in patients with asymptomatic internal-carotid artery stenosis. North American symptomatic carotid endarterectomy trial collaborators. N Engl J Med. 2000;342:1693-700.

13. Lee SJ, Cho SJ, Moon HS, Shon YM, Lee KH, Kim DI, et al. Combined extracranial and intracranial atherosclerosis in Korean patients. Arch Neurol. 2003;60:1561-4.

14. Zhao X, Underhill HR, Zhao Q, Cai J, Li F, Oikawa M, et al. Discriminating carotid atherosclerotic lesion severity by luminal stenosis and plaque burden: a comparison utilizing high-resolution magnetic resonance imaging at 3.0 tesla. Stroke. 2011;42:347-53.

15. Wasserman BA, Wityk RJ, Trout HH 3rd, Virmani R. Low-grade carotid stenosis: looking beyond the lumen with MRI. Stroke. 2005;36:2504-13.

16. Man BL, Fu YP, Chan YY, Lam W, Hui CF, Leung WH, et al. Use of magnetic resonance angiography to predict long-term outcomes of ischemic stroke patients with concurrent stenoses in Hong Kong. Cerebrovasc Dis. 2009;28:112-8,

17. Hoshino T, Sissani L, Labreuche J, Ducroca G, Lavallée PC, Meseguer E, et al. Prevalence of systemic atherosclerosis burdens and overlapping stroke etiologies and their associations with long-term vascular prognosis in stroke with intracranial atherosclerotic disease. JAMA Neurol. 2018;75:203-11.

18. Takaya N, Yuan C, Chu B, Saam T, Underhill H, Cai J, et al. Association between carotid plaque characteristics and subsequent ischemic cerebrovascular events: a prospective assessment with MRI-initial results. Stroke. 2006;37:818-23.

19. Kwee RM, van Oostenbrugge RJ, Mess WH, Prins MH, van der Geest RJ, ter Berg JW, et al. MRI of carotid atherosclerosis to identify TIA and stroke patients who are at risk of a recurrence. J Magn Reson Imaging. 2013;37:1189-94.

20. Zavodni AE, Wasserman BA, McClelland RL, Gomes AS, Folsom AR, Polak JF, et al. Carotid artery plaque morphology and composition in relation to incident cardiovascular events: the multi-ethnic study of atherosclerosis (MESA). Radiology. 2014;271:381-9.

21. Sun J, Zhao XQ, Balu N, Neradilek MB, Isquith DA, Yamada K, et al. Carotid plaque lipid content and fibrous cap status predict systemic CV outcomes: the MRI substudy in AIM-HIGH. JACC Cardiovasc Imaging. 2017;10:241-9.

22. Naghavi M, Libby P, Falk E, Casscells SW, Litovsky S, Rumberger J, et al. From vulnerable plaque to vulnerable patient: a call for new definitions and risk assessment strategies: part I. Circulation. 2003;108:1664-72.

23. Underhill HR, Yuan C, Yarnykh VL, Chu B, Oikawa M, Dong L, et al. Predictors of surface disruption with MR imaging in asymptomatic carotid artery stenosis. AJNR Am J Neuroradiol. 2010;31:487-93.

24. Xia J, Yin A, Li Z, Liu X, Peng X, Xie N. Quantitative analysis of lipid-rich necrotic Core in carotid atherosclerotic plaques by in vivo magnetic resonance imaging and clinical outcomes. Med Sci Monit. 2017;23:2745-50.

25. Glagov S, Weisenberg E, Zarins CK, Stankunavicius R, Kolettis GJ. Compensatory enlargement of human atherosclerotic coronary arteries. N Engl J Med. 1987;316:1371-5.

26. Zhou Z, Li R, Zhao X, He L, Wang X, Wang J, et al. Evaluation of 3D multicontrast joint intra- and extracranial vessel wall cardiovascular magnetic resonance. J Cardiovasc Magn Reson. 2015;17:41.

\section{Publisher's Note}

Springer Nature remains neutral with regard to jurisdictional claims in published maps and institutional affiliations. 